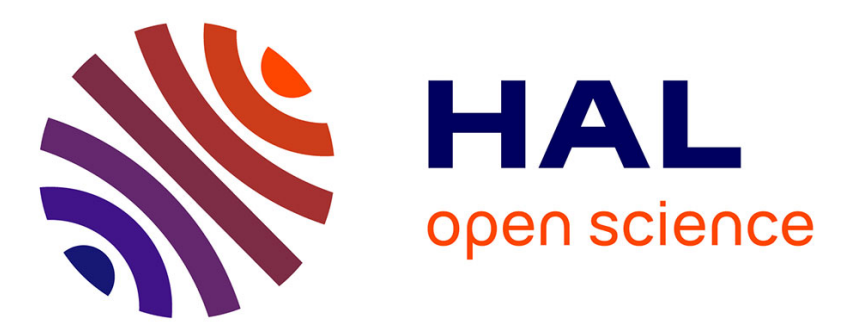

\title{
Shear Horizontal Phononic Metasurface for In-Liquid Gravimetric Biosensing
}

\author{
Gao Feng, Abdelkrim Khelif, Sarah Benchabane-Gaiffe, Amine Bermak
}

\section{To cite this version:}

Gao Feng, Abdelkrim Khelif, Sarah Benchabane-Gaiffe, Amine Bermak. Shear Horizontal Phononic Metasurface for In-Liquid Gravimetric Biosensing. IEEE Electron Device Letters, 2021, 42 (6), pp.915 - 918. hal-03385531

\author{
HAL Id: hal-03385531 \\ https://hal.science/hal-03385531
}

Submitted on 19 Oct 2021

HAL is a multi-disciplinary open access archive for the deposit and dissemination of scientific research documents, whether they are published or not. The documents may come from teaching and research institutions in France or abroad, or from public or private research centers.
L'archive ouverte pluridisciplinaire HAL, est destinée au dépôt et à la diffusion de documents scientifiques de niveau recherche, publiés ou non, émanant des établissements d'enseignement et de recherche français ou étrangers, des laboratoires publics ou privés. 


\title{
Shear Horizontal Phononic Metasurface for In-liquid Gravimetric Biosensing
}

\author{
Feng Gao, Member, IEEE, Abdelkrim Khelif, Sarah Benchabane, Amine Bermak, Fellow, IEEE
}

\begin{abstract}
In-liquid wave attenuation is the major factor that limits the resolution of acoustic wave biosensors. Existing acoustic wave devices rely on shear mode operation to reduce the mechanical motion transferred to the liquid. However, the energy leakage still exists through acoustic radiation. In this article, we combined phononic metasurface based on local mechanical resonators with shear horizontal surface acoustic wave (SH-SAW) to address the above issue. The shear-horizontal phononic metasurface (SH-PM) resonator is made of periodic high-aspectratio electrode (HARE). It produces a slow phononic wave that cannot propagate in water due to its low velocity, which suppresses the acoustic radiation. Furthermore, as the particle motion of the HARE is polarized in shear mode, the SH-PM also reduces the mechanical motion transfer to water. The minimization of the total acoustic energy leakage in the SH-PM results in high quality factor (Q) resonance in water. Besides, the increased surface-to-volume ratio of the HAREs also enhances the mass sensitivity of the device. Experimental results show that the SH-PM resonator achieved an in-liquid $Q$ factor of 230 and a normalized mass sensitivity of $192.4 \mathrm{~cm}^{2} / \mathrm{g}$. The high in-liquid $Q$ factor together with the high mass sensitivity makes the SH-PM a promising candidate for highresolution gravimetric detection of biomolecules.
\end{abstract}

Index Terms - Phononic metasurface, Biosensor, Surface Acoustic Wave, Gravimetric Sensing.

\section{Introduction}

The detection of biomolecules using acoustic biosensors is widely studied because of their high sensitivity, small size, lowcost and label-free characteristics ${ }^{1-3}$. However, acoustic biosensors suffer from strong in-liquid acoustic wave attenuation that reduces their quality factor (Q). The current approach for addressing this issue relies on shear mode operation of the acoustic wave devices to minimize mechanical motion transferred to the liquid environment ${ }^{4-6}$. Nevertheless, the shear polarization cannot prevent the energy leakage through acoustic radiation. To address this problem, phononic metasurface (PM) is introduced ${ }^{7,8}$.

PM resonator is formed by substituting the thin electrode in conventional surface acoustic wave (SAW) resonator with periodic high aspect ratio electrode (HARE) acting as local mechanical resonators. The acoustic wave produced in the PM has a velocity lower than the sound velocity in water, which

This work is funded by NPRP grant No. NPRP10-0201-170315 from the Qatar National Research Fund of Qatar Foundation. This work is also supported by the EIPHI Graduate School (contract "ANR-17-EURE-0002") and the FEMTO-ST technological facility of the French RENATECH network. The work also received funding from the European Research Council (ERC) under the European Union's Horizon 2020 research and innovation programme (grant agreement No. 865724)

Feng Gao and Amine Bermak are with the College of Science \& Engineering, HBKU, Doha, Qatar (e-mail: fgao@hbku.edu.qa; abermak@hbku.edu.qa;).

Abdelkrim Khelif, Sarah Benchabane are with FEMTO-ST Institute, CNRS, Besancon, France. (e-mail:abdelkrim.khelif@femto-st.fr; sarah.benchabane@femtost.fr.). Feng Gao is also with FEMTO-ST Institue. cannot propagate in water and thus eliminates the acoustic radiation. The previously reported vertically polarized PM (VP$\mathrm{PM}$ ) resonator derived from the classical Rayleigh waves demonstrated the radiation suppression phenomenon ${ }^{7}$. However, the vertical polarization together with the processinduced defects in the HARE compromised the suppression effect, leading to a limited $Q$ factor improvement. In this article, we designed a shear horizontal PM (SH-PM) resonator that combines the advantages of shear polarization and phononic metasurface, which can simultaneously suppress the energy leakage through acoustic radiation and mechanical motion transfer. In addition, the large surface-to-volume ratio of the HARE also enhances the mass sensitivity of SH-PM biosensor.

\section{Device Design and Fabrication}

Fig. 1a shows a top-view SEM image of the fabricated SHPM resonator. Thirty pairs of interdigitated transducers (IDTs) are located in the center of the device for generating the phononic wave, while 20 reflector strips are placed on the two sides of the IDTs for enhancing the resonance. The number of reflector strips used in the SH-PM resonator is significantly less than the one in conventional SAW resonators ${ }^{9}$. This is because the locally-resonant HARE produces phononic wave with very low group and phase velocities that suppress the energy propagation, and thus only a small amount of reflectors is needed for confining the acoustic energy. The pitch of the metasurface is $5 \mu \mathrm{m}$, which leads to a wavelength of $10 \mu \mathrm{m}$. The height of the HARE is $5.3 \mu \mathrm{m}$. The profile of the electrode is revealed by a tilted SEM image of the device in Fig. $1 \mathrm{~b}$.

The fabrication of the device is implemented by an electroplating-based process. 100-nm copper seed layer was firstly deposited on a four-inch Y-X lithium niobate wafer by sputtering. Optical photolithography is then used to create a 7$\mu \mathrm{m}$ thick photoresist mold for electroplating. Subsequently, 5.3- $\mu \mathrm{m}$ nickel layer is electroplated on the wafer surface to form the HARE. After stripping the photoresist mold, the copper seed layer between nickel structures is removed by reactive ion etching. At last, a 200-nm silicon dioxide layer is deposited and patterned to form a passivation layer for the IDTs and reflectors.

The SH-PM resonator achieves the prohibition of in-liquid wave propagation by producing an acoustic wave that is slower than the sound velocity in water through increasing the electrode height. Fig. 2 shows the phase velocity of the SH-PM resonator at different electrode heights simulated by finite element method (FEM). When the electrodes are higher than $3.7 \mu \mathrm{m}$, the phase velocity of the acoustic wave is slower than the sound velocity in water. 

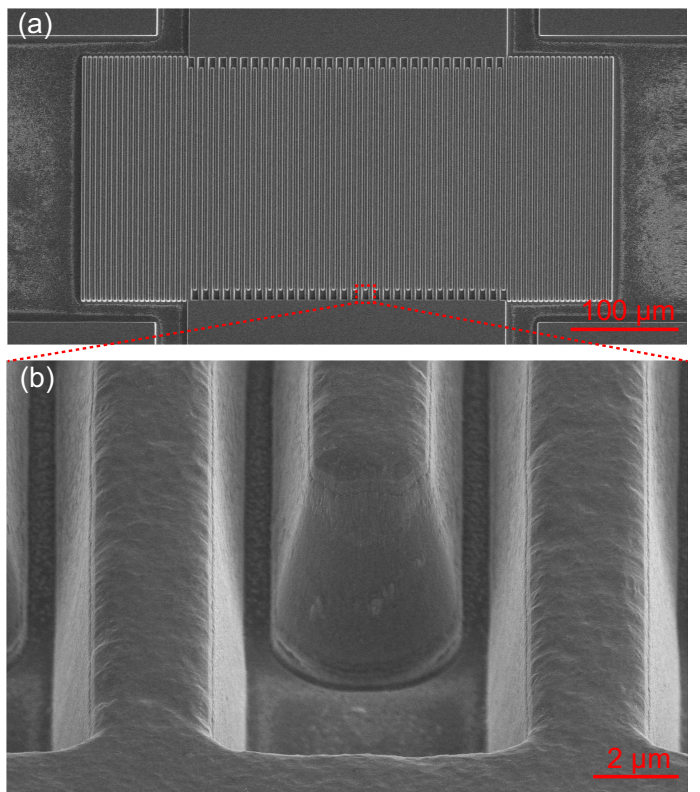

Figure 1. (a). Top-view SEM image of the SH-PM resonator. (b) Tilted SEM image of the HARE.

To reveal the phenomena of acoustic radiation suppression, the mode shapes and acoustic pressure distributions of two SHPM resonators and a conventional SH-SAW resonator immersed in water are simulated. Simplified unit cells are used in the simulation to reduce the computation time. Perfectly matched layers (PMLs) are exploited in the bottom of the substrate and top of the water to effectively extend their depths to infinity. Fig. 3a shows the simulation result for SH-PM with $5.3-\mu \mathrm{m}$ electrode. The positive and negative acoustic pressures in the water displayed by the red and blue regions distribute only near the electrodes, representing the local confinement of acoustic energy. In comparison, the PM resonator with $2-\mu \mathrm{m}$ electrode (Fig. 3b) and the SH-SAW (Fig. 3c) produces propagating acoustic waves in the water, which are seen by the alternating positive and negative acoustic pressures.

\section{Performance of the SH-PM resonator}

The mass sensing resolution of the SH-PM resonator is determined by its frequency noise divided by its mass sensitivity. Q factor is used to evaluate the frequency stability of the resonator. Fig. 4a shows the measured impedance of the SH-PM resonator in air and water, respectively. The minimum and maximum impedance points correspond to the resonance $\left(f_{r}\right)$ and anti-resonance frequencies $\left(f_{a}\right)$, respectively. The $\mathrm{Q}$ factor of the device is derived from its impedance by the following equation:

$$
Q=\frac{f}{2} \cdot\left|\frac{d \phi}{d f}\right|
$$

where $f$ and $\phi$ are the operating frequency and impedance phase of the device, respectively. The results in Fig. 4b show that the in-liquid Q factor of the SH-PM at $f_{a}$ deteriorated by only 1.34 times from 309 in air to 230 in water. For comparison, a conventional SH-SAW based on $36^{\circ}$-YX lithium tantalate operating at $417 \mathrm{MHz}$ was fabricated using the same layout. It was found that the Q factor of the SH-SAW at $f_{a}$ deteriorated

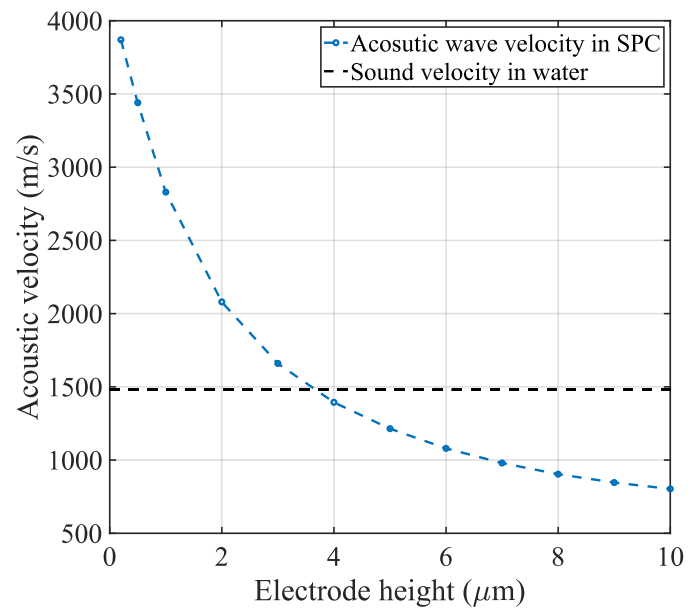

Fig. 2. Acoustic wave velocity of phononic metasurface with different electrode height in water

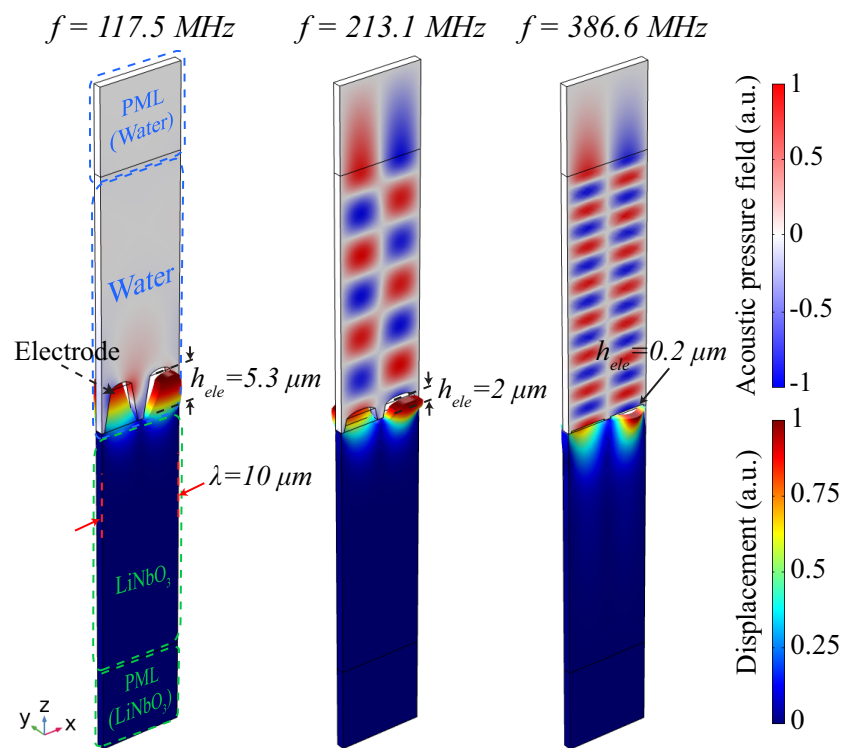

(a)

(b)

(c)

Fig. 3. Mode shape and acoustic pressure distribution of the SH-PM resonator with electrode heights of (a) $5.3 \mu \mathrm{m}$. (b). $2 \mu \mathrm{m}$ and (c) a regular SH-SAW resonator with thin electrodes.

by 4.58 times from 151 in air to 33 in water. The small relative Q factor degradation of SH-PM compared to SH-SAW proves the effectiveness of acoustic radiation suppression. The electromechanical coupling coefficient $\left(K^{2}=\pi^{2}\left(f_{a}-f_{r}\right) / 4 f_{r}\right)$ of the SH-PM in air and in water are $14.8 \%$ and $3.26 \%$, respectively. $K^{2}$ in water is lower than that in air due to the mismatch of dielectric constant between water and $\mathrm{LiNbO}_{3}$. The normalized mass sensitivity $\left(S_{m}=\Delta m /\left(\Delta f \cdot f_{r}\right)\right)$ of the SH-PM is characterized utilizing its electrode height variation across the wafer, which originates from the nonuniformity of the electroplating process. The height change is actually equivalent to the variation of additive mass on top of the electrode. The loaded mass causes variations in the elastic resonance of the HARE, the coupled SAW velocity and the electrode reflectivity, leading to a shift in the SH-PM resonance frequency. Fig. 5 shows the $f_{a}$ distribution with the variation of electrode height. The normalized SH-PM mass sensitivities 

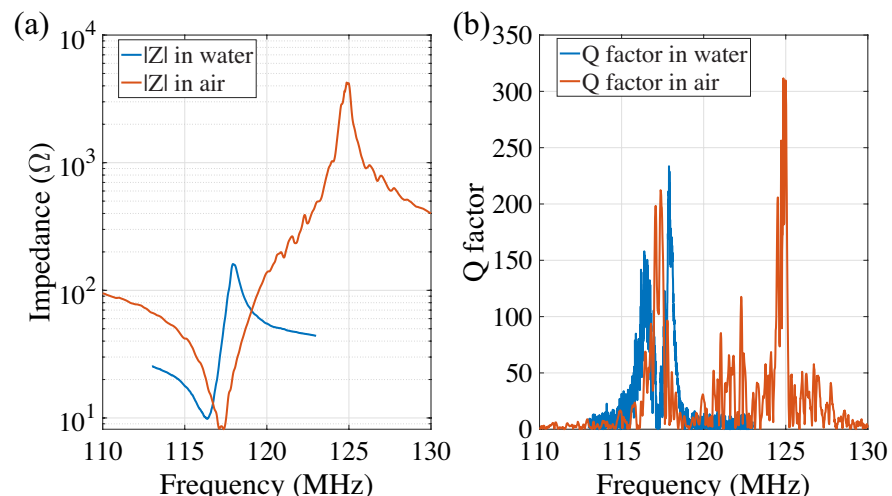

Fig. 4 Impedance (a) and Q factor (b) of the SH-PM resonator.

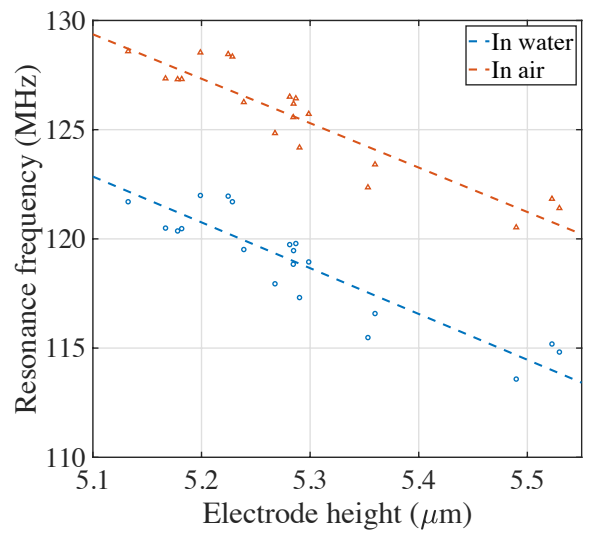

Fig. 5 Resonance frequency shift of the SH-PM resonator in response to electrode height variation.

Table 1. Comparison of SH-PM with other acoustic resonators

\begin{tabular}{|c|c|c|c|c|}
\hline Device & $\left|S_{m}\right|^{\mathrm{a}}$ & In-liquid Q & FOM & $f(\mathrm{MHz})$ \\
\hline SH-SAW & 99 & 3881 & $3.84 \times 10^{5}$ & 134.3 \\
S-FBAR & 3067.4 & 2574 & $7.90 \times 10^{6}$ & 1273.9 \\
HARE-LWR & 485.2 & $1.1 \times 10^{5}$ & $5.34 \times 10^{7}$ & 131.7 \\
SH-PM & 536.1 & $1.63 \times 10^{5}$ & $8.74 \times 10^{7}$ & 116.4 \\
\hline
\end{tabular}

${ }^{a}$ Unit of the normalized mass sensitivity, $S_{m}: \mathrm{cm}^{2} / \mathrm{g}$

are extracted from the slope of the linear fitted lines, which are $-184.3 \mathrm{~cm}^{2} / \mathrm{g}\left(R^{2}=0.84\right)$ and $-192.4 \mathrm{~cm}^{2} / \mathrm{g}\left(R^{2}=0.81\right)$ in air and in water, respectively. Compared to the previously reported VP-PM resonator ${ }^{7}$ that has an in-liquid Q factor of 50.4 and mass sensitivity of $-117.3 \mathrm{~cm}^{2} / \mathrm{g}$, the SH-PM resonator shows 4.6-time $\mathrm{Q}$ factor and 1.64-time mass sensitivity improvements, which represents an equivalent 7.5-time improvement of the overall sensing resolution.

We used FEM to compare the in-liquid performances of SHPM together with SH-SAW, shear mode film bulk acoustic wave resonator (S-FBAR) and the previously reported HARE Lamb wave resonator (HARE-LWR). We chose FEM to evaluate the $\mathrm{Q}$ factors and mass sensitivities because the experimental values are usually not reported. The simulation models of HARW-LWR and SH-SAW were previously reported in [8]. The simulated S-FBAR comprises of 2-um aluminum nitride (AIN) sandwiched by two $200-\mathrm{nm}$ aluminum electrodes. The c-axis of the AlN is titled by $30^{\circ}$ to support the excitation of shear mode. 100 -nm poly methyl methacrylate (PMMA) layer is used as the mass loading layer whose density is swept in the simulation to produce the frequency shift signal.
The simulation results in Table 1 show that SH-PM resonator exhibits the highest figure of merit $\left(F O M=S_{m} \times Q\right)$ among the four devices. The S-FBAR has the highest mass sensitivity due to its high operating frequency but a relatively low $\mathrm{Q}$ factor due to its in-liquid acoustic radiation. The simulated mass sensitivity of the S-FBAR is comparable to the reported mass sensitivity $\left(1690.1 \mathrm{~cm}^{2} / \mathrm{g}\right)$ of a $1.47-\mathrm{GHz}$ S-FBAR ${ }^{10}$. The mass sensitivities of SH-PM and HARE-LWR are significantly higher than the SH-SAW due to the high surface-to-volume ratio of HARE. They are also higher than the reported 272 $\mathrm{cm}^{2} / \mathrm{g}$ mass sensitivity of a conventional Lamb wave biosensor operating at $473 \mathrm{MHz}^{11}$, despite the later has a higher operating frequency. The simulated mass sensitivity of SH-PM is higher than the experimental values because the experiments only involves the mass loading on the top part of the HARE. As the adsorption of molecules in real applications are homogenous all-over device surface, a 200-nm PMMA layer uniformly covering the metasurface was used to obtain the homogenous loading mass sensitivity in simulation. Table 1 also shows that SH-PM has the highest $\mathrm{Q}$ factor among the four devices due to the simultaneous reduction of acoustic radiation and mechanical motion transfer. It should be noted that the simulated Q factors are much higher than the actual devices because the process and material defects are not considered in the simulation. Simulation without material damping enables the univariate modeling of acoustic radiation effect. Although material damping can be added to the simulation model, such artificial damping does not reflect the reality as the material defects are unknown. In addition to the high performance, SHPM also has low fabrication cost compared to S-FBAR and HARE-LWR because the later involve either a suspended film structure that requires back-etching or a distributed Bragg reflector that requires deposition of multiple layers of reflecting materials. Nevertheless, the defects in the electroplating process of the SH-PM is still a limiting factor for its Q value.

\section{Conclusion}

An SH-PM resonator is designed to overcome the in-liquid acoustic attenuation issue that limits the mass sensing resolution of acoustic wave biosensors. The PM was formed by electroplated nickel HAREs acting as mechanical resonators. It produces a surface-electrode hybridized locally-resonant wave with a velocity lower than the sound velocity in water, which does not propagate in water and thus fully stops the acoustic radiation. In addition, the shear horizontal polarization of the SH-PM reduces mechanical motion transferred to water, which further improves its Q factor. Experimental results showed the Q factor and mass sensitivity of the SH-PM resonator are 4.6 and 1.64 times higher than the previously reported VP-PM resonator, which would lead to an overall equivalent sensing resolution improvement of 7.5 times. A theoretical study through FEM also shows that the SH-PM is superior compared to other acoustic biosensors, including SH-SAW, S-FBAR, and HARE-LWR. In summary, the SH-PM resonator demonstrated a novel approach for high-resolution gravimetric biosensing through the combination of suppressed acoustic radiation enabled by the phononic metasurface and reduced mechanical motion transfer enabled by the shear horizontal polarization. 


\section{Reference}

1. K. Länge, "Bulk and Surface Acoustic Wave Sensor Arrays for Multi-Analyte Detection: A Review," Sensors, vol. 19, no. 24, pp. 5382, 2019. doi: 10.3390/s19245382.

2. Y. Zhang, J. Luo, A. J. Flewitt, Z. Cai and X. Zhao, "Film bulk acoustic resonators (FBARs) as biosensors: A review," Biosens. Bioelectron., vol. 116, pp. 1-15, 2018. doi: 10.1016/j.bios.2018.05.028.

3. N. G. Durmuş, R. L. Lin, M. Kozberg, D. Dermici, A. Khademhosseini and U. Demirci, "Acoustic-based biosensors," Encycl. Microfluid. Nanofluid., pp. 28-40, 2015. doi: 10.1007/978-3-642-27758-0_10-2.

4. S. Song, D. Chen, H. Wang, C. Li, W. Wang, W. Yu, Y. Wang and Q. Guo, "Shear mode bulk acoustic resonator based on inclined c-Axis AlN film for monitoring of human hemostatic parameters," Micromachines, vol. 9, no. 10, pp. 501, 2018. doi: $10.3390 / \mathrm{mi} 9100501$.

5. J. Ji, Y. Pang, D. Li, Z. Huang, Z. Zhang, N. Xue, Y. Xu and $X$. Mu, "An aptamer-based shear horizontal surface acoustic wave biosensor with a CVD-grown single-layered graphene film for high-sensitivity detection of a label-free endotoxin," Microsyst. Nanoeng., vol. 6, no. 1, pp. 1-11, 2020. doi: 10.1038/s41378-019-0118-6.

6. J. Ji, C. Yang, F. Zhang, Z. Shang, Y. Xu, Y. Chen, M. Chen and $\mathrm{X}$. Mu, "A high sensitive SH-SAW biosensor based $36^{\circ} \mathrm{YX}$ black $\mathrm{LiTaO} 3$ for label-free detection of Pseudomonas Aeruginosa," Sens. Actuators, B, vol. 281, pp. 757-764, 2019. doi: 10.1016/j.snb.2018.10.128.

7. F. Gao, A. Bermak, S. Benchabane, L. Robert and A. Khelif, "Acoustic radiation-free surface phononic crystal resonator for in-liquid low-noise gravimetric detection," Microsyst. Nanoeng., vol. 7, no. 1, pp. 8, 2021 . doi: 10.1038/s41378-02000236-9.

8. F. Gao, A. M. Al-Qahtani, A. Khelif, F. Boussaid, S. Benchabane, Y. Cheng, O. El Agnaf and A. Bermak, "Towards Acoustic Radiation Free Lamb Wave Resonators for Highresolution Gravimetric Biosensing," IEEE Sens. J., vol. 21, no. 3, pp. 2725-2733, 2021. doi: 10.1109/JSEN.2020.3023854.

9. D. Morgan, Surface acoustic wave filters: With applications to electronic communications and signal processing. Academic Press, 2010, doi: 10.1016/B978-0-12-372537-0.X5000-6.

10. T. Mirea, M. Clement, J. Olivares and E. Iborra, "Assessment of the Absolute Mass Attachment to an AlNBased Solidly Mounted Resonator Using a Single Shear Mode," IEEE Electron Device Letters, vol. 41, no. 4, pp. 609-612, 2020. 11. X. Lu, C. M. Lee, S. Y. Wu, H. P. Ho and K. M. Lau, "GaNBased S0-Wave Sensors on Silicon for Chemical and Biological Sensing in Liquid Environments," IEEE Sens. J., vol. 13, no. 4, pp. 1245-1251, 2012. 